\title{
Effects of Three Different Methods Defining Onset of Peripheral Artery Disease on the Assessments of Incidence and Important Predictors - Results from the German Epidemiological Trial on Ankle Brachial Index (getABI)
}

\author{
Alexander Lupilov \\ Dietmar Krause (iD) \\ Renate Klaassen-Mielke \\ Hans J Trampisch (D) \\ Henrik Rudolf (iD) \\ Department of Medical Informatics, \\ Biometry, and Epidemiology, Ruhr \\ University Bochum, Bochum, North- \\ Rhine-Westphalia, Germany
}

Purpose: The common definition of asymptomatic peripheral artery disease (PAD) by a single determination of the ankle brachial index (ABI) has some uncertainty due to measurement errors. This may impact estimates of PAD incidence and assessment of PAD risk factors. To investigate this issue, we used three methods to define asymptomatic PAD and made use of data from the German Epidemiological Trial on Ankle Brachial Index (getABI).

Patients and Methods: A total of 6,880 unselected subjects aged $\geq 65$ years, enrolled by 344 trained general practitioners, had ABI assessments at baseline and four visits during follow-up. The first approach defined asymptomatic PAD onset as soon as a single ABI value was below 0.9 (single ABI). The second approach employed a regression method using all available ABI values (regression A), while for the third approach (regression B), an extended regression beyond the last valid $\mathrm{ABI}$ value for the observation time of the study was allowed. For each approach, we calculated PAD incidence rates and assessed the effect of important PAD predictors using multivariable Cox proportional hazards regression.

Results: The regression method A showed the lowest (25.0 events per 1,000 person years) and the single ABI method the highest incidence rate (41.2). The regression methods assigned greater impact to several risk factors of incident PAD. Using regression A, the hazard ratios (HR) of active smoking $(2.36 ; 95 \%$ CI 1.92 to 2.90$)$ and of diabetes $(1.33 ; 95 \%$ CI 1.13 to 1.56$)$, using regression $B$ the HR of older age (1.72; 95\% CI 1.50 to 1.97$)$ were about twice as high as the corresponding HR of the single ABI approach.

Conclusion: Use of the single ABI method leads to higher PAD incidence rates and to lower impact of important PAD predictors compared to regression methods. For an alert risk factor management, multiple ABI determination may be useful.

Keywords: peripheral artery disease, ankle brachial index, risk factors, incidence

\section{Plain Language Summary}

The asymptomatic peripheral artery disease (PAD) is commonly diagnosed by the ankle brachial index (ABI), in case it is below 0.9. The ABI is a value calculated from measurements of the blood pressure at the limbs. There might be some uncertainty in diagnosis due to measurement errors. In our study, the ABI was determined several times per participant in the follow-up of seven years. We wanted to find out, if other definitions of asymptomatic PAD using more than one ABI value would change the number of new diagnoses in the
Correspondence: Henrik Rudolf Department of Medical Informatics, Biometry, and Epidemiology, Ruhr University Bochum, Universitaetsstrasse 105, Bochum, 44789, Germany

Tel +492343221494

Fax +492343214325

Email henrik.rudolf@rub.de 
cohort. Beyond the single ABI method, we introduce two methods, which determine a linear trend for each participant from all his/her ABIs by so-called linear regression. For all three approaches we estimated the rate of newly developed PAD. Hereby, one approach (regression A) used the estimated trend only inside the timepoints of valid ABI assessments, whereas the other (regression B) allowed the trend to be extended until the end of the study. Further, we investigated how risk factors for PAD onset changed.

\section{Introduction}

Peripheral artery disease (PAD) is an increasingly important public health problem, affecting over 236 million people globally. ${ }^{1}$ Symptomatic PAD can become apparent through intermittent claudication, critical limb ischemia, the need for revascularization or limb amputation. ${ }^{2}$ Asymptomatic PAD can be determined by the ankle brachial index (ABI). ${ }^{3}$ Both are associated with increased risk of cardiovascular events and premature death. ${ }^{4,5}$ Previous studies on PAD incidence and associated risk factors had shown considerably varying incidence rates ranging between 3.8 and 23.8 events per 1,000 person years. ${ }^{6,7}$ The wide range of results might not only depend on the analyzed cohort, but also on the method to define PAD onset. To investigate this issue, we used three different approaches to define asymptomatic PAD. Beyond the common approach using a single ABI value lower than 0.9 (here referred as single $\mathrm{ABI}$ method), ${ }^{8}$ we set up two regression methods (A, B) to assess asymptomatic PAD, using data from the German Epidemiological Trial on Ankle Brachial Index (getABI). ${ }^{9}$ The aim of our work was to compare these three approaches regarding PAD incidence rates and the importance of conventional and novel PAD predictors for time to PAD onset.

\section{Patients and Methods}

\section{German Epidemiological Trial on Ankle Brachial Index (getABI)}

The German Epidemiological Trial on Ankle Brachial Index was launched in October 2001 as a prospective cohort study to collect data on PAD incidence as well as important PAD predictors. Three hundred and forty-four general practitioners (GP) from all regions in Germany enrolled a total of 6,880 unselected participants. ${ }^{4}$ The baseline examination included medical and drug history, laboratory diagnostics and a physical examination with determination of the ABI values. They were calculated from values of Doppler sonography blood pressure measurements at upper and lower limbs. After 12, 36, 60, and 84 months, follow-up examinations were performed. ${ }^{9}$

\section{Three Different Approaches to Assess Asymptomatic PAD}

Each definition of PAD comprises a symptomatic and an asymptomatic part. Symptomatic PAD was characterized by intermittent claudication, peripheral revascularization, necrosis/gangrene, or amputation. For the asymptomatic part, beyond the single ABI method (ABI value below 0.9 ), we applied two regression methods. In regression method A, we used all time points of valid ABI assessments per patient over the course of the getABI study to determine a regression line. If the regression line intersected the horizontal level of 0.9 before the last ABI, this intersection point was defined as PAD onset. ${ }^{2}$ In the regression method $\mathrm{B}$, we used the same regression line, but allowed an extension beyond the last ABI value until the end of the observation period. Please note that regression methods $\mathrm{A}$ and $\mathrm{B}$ do not differ in participants with a valid $\mathrm{ABI}$ assessment at the last follow-up after seven years.

For these three approaches to assess asymptomatic PAD, we introduce the notation "PAD definition" for the combination of symptomatic PAD with asymptomatic PAD by either single ABI method (PAD definition 1), regression method $\mathrm{A}$ (PAD definition 2), or regression method B (PAD definition 3).

If subjects with an $\mathrm{ABI}$ value $>1.5$ at baseline did not show any PAD symptoms, they were excluded from the analysis due to suspected media sclerosis. Subjects without PAD at baseline were included in this post hoc study on PAD incidence and the assessment of PAD predictors. The PAD-free cohort comprised all participants without symptoms and an $\mathrm{ABI} \geq 0.9$ and a regression intercept $\geq 0.9$ at baseline. Although this definition is only feasible in posthoc analysis but not in clinical practice (because it uses values from the future), it was necessary for the sake of good comparability of all PAD definitions starting from the same PAD-free cohort. Time until PAD onset, time until death or time until the end of the observation period was used as the time at risk.

\section{Definitions of Risk Factors}

Our choice of risk factors was based on previous publications of the getABI study group. ${ }^{2}$ These were age, sex, active smoking status, arterial hypertension, diabetes, lowdensity lipoprotein (LDL), lipid lowering medication, body-mass-index (BMI), pre-existing cardiovascular or cerebrovascular disease, education (classified by the 
International Standard Classification of Education, ISCED), homocysteine (HCY), C-reactive protein (CRP), gamma-glutamyl transferase (GGT), estimated glomerular filtration rate (eGFR), and vitamin D.

All risk factors were assessed at baseline, missing values were imputed by randomly selected values of the full getABI cohort. Dichotomization of factors was done according to common thresholds or using the median. Other categorizations were used for age ( $>75$ years vs $\leq 75$ years), arterial hypertension (positive if diagnosed by the treating physician or in case of use of angiotensin-1 receptor antagonists or angiotensin converting enzyme (ACE) inhibitors or diuretics), lipid lowering therapy (positive in case of statin or fibrate use), pre-existing cardiovascular or cerebrovascular disease (history of heart attack, stroke, coronary revascularization, or carotid revascularization), education according to ISCED (low education level: ISCED 0-2 vs normal or high education level: ISCED 3-6), GGT (the highest GGT quartile vs other, $>18$ $\mathrm{U} / \mathrm{L}$ vs $\leq 18 \mathrm{U} / \mathrm{L}$ for female sex and $>26 \mathrm{U} / \mathrm{L}$ vs $\leq 26 \mathrm{U} / \mathrm{L}$ for male sex).

\section{Primary Outcome Measure}

The primary outcome measure was time to PAD onset according to the respective method defining PAD onset.

\section{Statistical Methods}

First, we investigated the characteristics of participants without signs of PAD at baseline and compared them with the complete set of getABI participants. Next, for each of the three PAD definitions, incidence rates were calculated as events per 1,000 person years. Then, univariable and multivariable Cox proportional hazard regression was done for the time to PAD onset, incorporating the 15 pre-selected risk factors. For each PAD definition a model was fitted. $\mathrm{P}$ values $<0.05$ were considered statistically significant. All $\mathrm{p}$ values are two-sided. Data analysis was performed using SAS software, version 9.4 (2013, SAS Insitute Inc., Cary, North Carolina, USA). The getABI trial was approved by the institutional review board of the University of Heidelberg and conducted in accordance with the guidelines of the German Association of Epidemiology for good epidemiological practice. The getABI trial was supported by unrestricted grants from Sanofi-Aventis GmbH, Berlin, Germany, and the German Federal Ministry of Education and Research. Data of the getABI trial is not freely available. All participants presented written informed consent before entering. This post-hoc analysis was reviewed and approved by the ethics committee of the Ruhr University Bochum (registration number: 16-5908). Trial registration: DRKS00011623.

\section{Results}

\section{Baseline Characteristics}

From the 6,880 subjects of the getABI study, we excluded 58 due to media sclerosis. Of the remaining 6,822 patients $30.7 \%$ were $>75$ years old, $9.3 \%$ were active smokers, $25.6 \%$ had diabetes, $16 \%$ had pre-existing cardiovascular or cerebrovascular disease and $19.6 \%$ had an eGFR $<60 \mathrm{~mL} / \mathrm{min} / 1.73 \mathrm{~m}^{2}$. $58 \%$ were women (Table 1, column 1).

The PAD-free group at baseline comprised 5,269 participants. In general, the PAD-free group at baseline had lower frequencies of risk factors compared to the whole cohort (Table 1, column 2). Differences were particularly pronounced in the risk factors age $>75$ years $(27.4 \%$ vs $30.7 \%$ ), active smoking status (7.8\% vs 9.3\%), diabetes (22.6\% vs $25.6 \%$ ), pre-existing cardiovascular or cerebrovascular diseases (12.9\% vs $16 \%$ ) and eGFR $<60 \mathrm{~mL} / \mathrm{min} /$ $1.73 \mathrm{~m}^{2}$ (16.9\% vs $\left.19.6 \%\right)$.

\section{PAD Incidence}

Out of 5,269 participants without signs of PAD at baseline, there were 313 (5.9\%) who experienced a PAD symptom, and 1,101 (20.9\%) had at least one ABI below 0.9 in seven years of follow-up. Due to regression A 536 (10.2\%) had asymptomatic PAD onset and 868 (16.5\%) due to regression B. During the maximum observation period of 7.25 years, 1,270 study participants experienced PAD onset according to the 1 st PAD definition (single ABI). Incidence rate was 41.2 (95\% CI 38.95 to 43.49 , Table 2) events per 1,000 person years. The 2 nd definition (regression A) with 810 new PAD cases displayed the lowest incidence rate at 25.0 (95\% CI 23.32 to 26.77) events per 1,000 person years among all three definitions. The incidence rate according to the 3rd definition (regression B) was in between (Table 2).

\section{Risk Factors}

In the univariable analysis, the risk factors age $>75$ years, active smoking status, arterial hypertension, diabetes, preexisting cardio- or cerebrovascular disease, body mass index (BMI) $\geq 30 \mathrm{~kg} / \mathrm{m}^{2}$, low ISCED (0-2), elevated homocysteine (HCY > median), CRP $>3 \mathrm{mg} / \mathrm{L}$, GGT highest quartile, low renal function $\left(\mathrm{eGFR}<60 \mathrm{~mL} / \mathrm{min} / 1.73 \mathrm{~m}^{2}\right.$ ), and vitamin 
Table I Baseline Characteristics of PAD-free Group in Comparison to the Cohort

\begin{tabular}{|c|c|c|c|}
\hline & & $\begin{array}{l}\text { All Patients } \\
\text { N(\%) }\end{array}$ & $\begin{array}{l}\text { PAD-free Patients } \\
\text { at Baseline N(\%) }\end{array}$ \\
\hline \multicolumn{2}{|l|}{ Total } & $6,822(100)$ & $5,269(100)$ \\
\hline \multicolumn{2}{|c|}{ Age $>75$ years } & $2,091(30.7)$ & $\mathrm{I}, 445(27.4)$ \\
\hline \multirow[t]{2}{*}{ Sex } & Male & $2,863(42.0)$ & $2,145(40.7)$ \\
\hline & Female & $3,959(58.0)$ & $3,124(59.3)$ \\
\hline \multicolumn{2}{|c|}{ Active smoking status } & $634(9.3)$ & $409(7.8)$ \\
\hline \multicolumn{2}{|c|}{ Arterial hypertension } & $4,735(69.4)$ & $3,446(65.4)$ \\
\hline \multicolumn{2}{|l|}{ Diabetes } & $\mathrm{I}, 744(25.6)$ & I, $188(22.6)$ \\
\hline \multirow[t]{2}{*}{$\begin{array}{l}\text { Lipid } \\
\text { metabolism }\end{array}$} & $\begin{array}{l}\mathrm{LDL} \\
\geq 130 \mathrm{mg} / \mathrm{dL}\end{array}$ & $2,921(42.8)$ & $2,290(43.5)$ \\
\hline & $\begin{array}{l}\text { Lipid lowering } \\
\text { medication }\end{array}$ & $\mathrm{I}, 607(23.6)$ & $\mathrm{I}, 146(2 \mathrm{I} .8)$ \\
\hline \multicolumn{2}{|c|}{$\mathrm{BMI} \geq 30 \mathrm{~kg} / \mathrm{m}^{2}$} & I,575 (23.1) & $\mathrm{I}, 188(22.6)$ \\
\hline \multicolumn{2}{|c|}{$\begin{array}{l}\text { Pre-existing cardio- or } \\
\text { cerebrovascular disease }\end{array}$} & $1,091(16.0)$ & $681(12.9)$ \\
\hline \multicolumn{2}{|c|}{ Low ISCED (0-2) } & I,698 (24.9) & I,272 (24.I) \\
\hline \multicolumn{2}{|c|}{$\mathrm{HCY}>$ median } & $3,419(50.1)$ & $2,514(47.7)$ \\
\hline \multicolumn{2}{|c|}{$\mathrm{CRP}>3 \mathrm{mg} / \mathrm{L}$} & $2,642(38.7)$ & $1,918(36.4)$ \\
\hline \multicolumn{2}{|c|}{ GGT highest quartile } & $\mathrm{I}, 676(24.6)$ & $\mathrm{I}, 243(23.6)$ \\
\hline \multicolumn{2}{|c|}{ eGFR $<60 \mathrm{~mL} / \mathrm{min} / 1.73 \mathrm{~m}^{2}$} & $1,337(19.6)$ & 891 (16.9) \\
\hline \multicolumn{2}{|c|}{ Vitamin $D<50 \mathrm{nmol} / \mathrm{L}$} & 4,741 (69.5) & $3,574(67.8)$ \\
\hline
\end{tabular}

Abbreviations: PAD, peripheral artery disease; LDL, Low density lipoprotein; BMI, body mass index; ISCED, International Standard Classification of Education; HCY, homocysteine; CRP, C-reactive protein; GGT, gamma-glutamyl transferase; eGFR, estimated glomerular filtration rate.

$\mathrm{D}<50 \mathrm{nmol} / \mathrm{L}$ were all significant PAD predictors according to all three PAD definitions (Table 3).

In the multivariable analysis, $\mathrm{HCY}>$ median remained significant according to the 1st PAD definition, and not to the 2 nd and 3 rd. Contrary to the 1 st and 2 nd definition, the 3rd definition exploits the risk factor low vitamin $\mathrm{D}(<50$ $\mathrm{nmol} / \mathrm{L}, 1.16,95 \% \mathrm{CI} 1.01$ to 1.33 ) as significant in the multivariable analysis. Low eGFR and GGT highest quartile were not significantly associated with incident PAD according to all three PAD definitions any longer (Table 4).

The estimated hazard ratios (HR) of many risk factors differed notably depending on the used PAD definition, especially in the multivariable analysis. The 1st PAD definition which used the single ABI method ascribed many PAD predictors a lower importance compared to the 2 nd and 3rd definition, which used regression methods. According to the 2nd PAD definition, the effect of the risk factors age $>75$ years (HR 1.66, 95\% CI 1.42 to 1.95), active smoking (HR 2.36, 95\% CI 1.92 to 2.90), and diabetes (HR 1.33, 95\% CI 1.13 to 1.56 ) on incident PAD were about twice as high compared to the 1st PAD definition. Comparing the two regression methods (PAD definition 2 and 3), estimates were considerably similar for most of the risk factors under investigation.

\section{Discussion Overview}

Within the scope of this post-hoc analysis of the largescale getABI study, we used three different approaches to assess asymptomatic PAD and investigated their impact on the assessment of PAD incidence and estimation of conventional as well as novel risk factors. The regression method A was introduced in Krause et al. ${ }^{2}$ The extension of regression lines in regression method $\mathrm{B}$ beyond its domain of time of known ABI values (extrapolation) might be questionable in statistical terms. On the other hand, PAD progresses with time, and of note missing values in epidemiological cohorts frequently appear from patients in average poorer condition.

Our results yield hints for overestimation of PAD incidence by single ABI method. It is well known that standard deviation of ABI values is about $8-9 \%{ }^{8}$ That means that ABI values below 0.9 may appear by chance. In

Table 2 Number of Events, Person Years, and Event Rates

\begin{tabular}{|l|c|c|c|c|}
\hline PAD Definition & $\begin{array}{c}\text { Number of } \\
\text { Events }\end{array}$ & $\begin{array}{c}\text { Median Time Until PAD } \\
\text { Onset (Years) }\end{array}$ & $\begin{array}{c}\text { Time Under Risk (Sum of } \\
\text { Person Years) }\end{array}$ & $\begin{array}{c}\text { Events per I,000 Person } \\
\text { Years (95\% Cl) }\end{array}$ \\
\hline Single ABI method & 1,270 & 3.0 & 30,810 & $41.2(38.95-43.49)$ \\
Regression method A & 810 & 2.9 & 32,349 & $25.0(23.32-26.77)$ \\
Regression method B & 1,077 & 3.4 & 31,732 & $33.9(31.91-35.97)$ \\
\hline
\end{tabular}

Abbreviations: PAD, peripheral artery disease; $\mathrm{Cl}$, confidence interval. 
Table 3 Association Between Risk Factors at Baseline and PAD Incidence, Univariable Analysis

\begin{tabular}{|c|c|c|c|c|c|c|c|}
\hline \multirow{2}{*}{\multicolumn{2}{|c|}{ Risk factors }} & \multicolumn{6}{|c|}{ HR (95\% Cl); p value } \\
\hline & & \multicolumn{2}{|c|}{$\begin{array}{l}\text { Ist PAD definition } \\
\text { (Single ABI) }\end{array}$} & \multicolumn{2}{|c|}{$\begin{array}{l}\text { 2nd PAD definition } \\
\text { (Regression A) }\end{array}$} & \multicolumn{2}{|c|}{$\begin{array}{l}\text { 3rd PAD definition } \\
\text { (Regression B) }\end{array}$} \\
\hline \multicolumn{2}{|l|}{ Age $>75$ years } & $1.34(1.19-1.51)$ & $<0,00$ I & $1.68(1.45-1.94)$ & $<0.001$ & $1.81(1.60-2.05)$ & $<0.001$ \\
\hline \multicolumn{2}{|l|}{ Male sex } & $1.05(0.96-1.15)$ & 0.283 & $1.28(1.15-1.42)$ & $<0.001$ & $1.16(1.05-1.28)$ & 0.003 \\
\hline \multicolumn{2}{|c|}{ Active smoking status } & $1.72(1.49-1.98)$ & $<0,001$ & $1.92(1.65-2.25)$ & $<0.001$ & $1.80(1.55-2.09)$ & $<0.001$ \\
\hline \multicolumn{2}{|c|}{ Arterial hypertension } & $1.52(1.38-1.68)$ & $<0.001$ & $1.54(1.37-1.73)$ & $<0.001$ & $1.47(1.32-1.63)$ & $<0.001$ \\
\hline \multicolumn{2}{|l|}{ Diabetes } & $1.42(1.28-1.57)$ & $<0.001$ & $1.54(1.38-1.72)$ & $<0.001$ & $1.52(1.36-1.69)$ & $<0.001$ \\
\hline \multirow[t]{2}{*}{ Lipid metabolism } & $\mathrm{LDL} \geq 130 \mathrm{mg} / \mathrm{dL}$ & $0.99(0.91-1.09)$ & 0.883 & $0.92(0.83-1.02)$ & 0.112 & $0.95(0.86-1.05)$ & 0.313 \\
\hline & Lipid lowering medication & $1.03(0.93-1.15)$ & 0.576 & $0.96(0.85-1.09)$ & 0.506 & $0.92(0.82-1.04)$ & 0.171 \\
\hline \multicolumn{2}{|l|}{$\mathrm{BMI} \geq 30 \mathrm{~kg} / \mathrm{m}^{2}$} & $1.20(1.08-1.33)$ & $<0.001$ & $1.14(1.01-1.28)$ & 0.030 & $1.17(1.05-1.31)$ & 0.005 \\
\hline \multicolumn{2}{|c|}{ Pre-existing cardiovascular or cerebrovascular disease } & $1.60(1.42-1.80)$ & $<0.001$ & $1.85(1.63-2.1 \mathrm{I})$ & $<0.001$ & $1.70(1.50-1.93)$ & $<0.001$ \\
\hline \multicolumn{2}{|l|}{ Low ISCED (0-2) } & $1.31(1.18-1.44)$ & $<0.001$ & $1.27(1.14-1.43)$ & $<0.001$ & $1.32(1.19-1.47)$ & $<0.001$ \\
\hline \multicolumn{2}{|l|}{$\mathrm{HCY}>$ median } & $1.38(1.26-1.52)$ & $<0.001$ & $1.47(1.32-1.62)$ & $<0.001$ & $1.38(1.25-1.52)$ & $<0.001$ \\
\hline \multicolumn{2}{|l|}{ CRP $>3 \mathrm{mg} / \mathrm{L}$} & $1.30(1.19-1.43)$ & $<0.001$ & $1.38(1.25-1.53)$ & $<0.001$ & $1.37(|.24-| .5 I)$ & $<0.001$ \\
\hline \multicolumn{2}{|c|}{ GGT highest quartile } & $1.22(1.10-1.35)$ & $<0.001$ & $1.30(1.16-1.45)$ & $<0.001$ & $1.25(1.12-1.39)$ & $<0.001$ \\
\hline \multicolumn{2}{|c|}{ eGFR $<60 \mathrm{~mL} / \mathrm{min} / 1.73 \mathrm{~m}^{2}$} & $1.61(1.45-1.79)$ & $<0.001$ & $1.85(1.64-2.08)$ & $<0.001$ & $1.82(1.63-2.04)$ & $<0.001$ \\
\hline \multicolumn{2}{|c|}{ Vitamin $D<50 \mathrm{nmol} / \mathrm{L}$} & $1.39(1.26-1.54)$ & $<0.001$ & $1.40(1.25-1.58)$ & $<0.001$ & $1.44(1.29-1.61)$ & $<0.001$ \\
\hline
\end{tabular}

Abbreviations: PAD, peripheral artery disease; LDL, Low density lipoprotein; BMI, body mass index; ISCED, International Standard Classification of Education; HCY, homocysteine; CRP, C-reactive protein; GGT, gamma-glutamyl transferase; eGFR, estimated glomerular filtration rate; $\mathrm{Cl}$, confidence interval; $\mathrm{N}$, number.

contrast, the standard error of prediction via regression is lower (up to a factor of $\sqrt{ } \mathrm{n}$, where $\mathrm{n}$ is the number of measurements). Compared to a single value below 0.9, the regression line can be interpreted as a means to minimize measurement errors. Therefore, the regression methods can be assumed to yield a more precise estimation of PAD onset.

The disparity of PAD incidence rates between the 1st PAD definition which used a single ABI value below 0.9 to define asymptomatic PAD onset and those using regression methods was large. The 1st PAD definition displayed the highest PAD incidence rate (41.2 events per 1000 person years). The 2nd PAD definition (regression A) contributed the lowest incidence rate (25.0). This might be an underestimation, because $A B I$ values are not missing at random and missing values may reflect poorer condition of such participants. Even after the extension of the individual regression lines beyond the time of the last ABI assessment (3rd PAD definition, regression B), incidence rates remained notably lower 33.9 (95\% CI 31.91 to 35.97) compared to the 1st PAD definition with the single ABI method. Probably the third definition combines the advantages of lower (measurement) errors and containing patients with poorer conditions, and therefore might be preferable for theoretical reasons. This of course would have to be checked in further studies, eg, by comparing the discriminatory capabilities of the definitions for endpoints such as mortality.

The estimates of many conventional as well as novel PAD risk factors varied greatly according to different definitions. The 1st PAD definition (single ABI) generally ascribed PAD predictors a lesser effect on PAD development compared to the 2nd (regression A) and 3rd (regression B) definition. This might reflect the greater measurement error in the single ABI method, blurring the risk factor estimation. Especially great differences were seen for the HRs of the risk factors age (1.28 vs 
Table 4 Association Between Risk Factors at Baseline and PAD Incidence, Multivariable Analysis

\begin{tabular}{|c|c|c|c|c|c|c|c|}
\hline \multirow{2}{*}{\multicolumn{2}{|c|}{ Risk factors }} & \multicolumn{6}{|c|}{ HR (95\% Cl); p value } \\
\hline & & \multicolumn{2}{|c|}{$\begin{array}{l}\text { Ist PAD definition } \\
\text { (Single ABI) }\end{array}$} & \multicolumn{2}{|c|}{$\begin{array}{c}\text { 2nd PAD definition } \\
\text { (Regression A) }\end{array}$} & \multicolumn{2}{|c|}{$\begin{array}{l}\text { 3rd PAD definition } \\
\text { (Regression B) }\end{array}$} \\
\hline \multicolumn{2}{|l|}{ Age $>75$ years } & $1.28(1.12-1.46)$ & $<0.001$ & $1.66(1.42-1.95)$ & $<0.001$ & $1.72(1.50-1.97)$ & $<0.001$ \\
\hline \multicolumn{2}{|l|}{ Male sex } & $0.89(0.78-1.01)$ & 0.068 & $1.10(0.94-1.29)$ & 0.235 & $1.06(0.92-\mid .2 I)$ & 0.450 \\
\hline \multicolumn{2}{|c|}{ Active smoking status } & $1.79(1.50-2.15)$ & $<0.001$ & $2.36(1.92-2.90)$ & $<0.001$ & $2.08(\mid .72-2.5 I)$ & $<0.001$ \\
\hline \multicolumn{2}{|c|}{ Arterial Hypertension } & $1.37(1.21-1.56)$ & $<0.001$ & $1.38(1.17-1.63)$ & $<0.001$ & $1.23(|.07-| .4 I)$ & 0.004 \\
\hline \multicolumn{2}{|l|}{ Diabetes } & $1.20(1.06-1.37)$ & 0.006 & $1.33(1.13-1.56)$ & $<0.001$ & $1.33(1.16-1.53)$ & $<0.001$ \\
\hline \multirow[t]{2}{*}{ Lipid metabolism } & $\mathrm{LDL} \geq 130 \mathrm{mg} / \mathrm{dL}$ & $1.23(1.10-1.38)$ & $<0.001$ & $1.25(1.08-1.44)$ & 0.002 & $1.15(1.02-1.30)$ & 0.025 \\
\hline & Lipid lowering medication & $1.12(0.98-1.28)$ & 0.089 & $1.04(0.88-1.23)$ & 0.646 & $0.95(0.82-I .1 I)$ & 0.526 \\
\hline \multicolumn{2}{|l|}{$\mathrm{BMI} \geq 30 \mathrm{~kg} / \mathrm{m}^{2}$} & $1.17(1.03-1.34)$ & 0.020 & $1.19(1.01-1.40)$ & 0.041 & $1.19(1.03-1.37)$ & 0.020 \\
\hline \multicolumn{2}{|c|}{ Pre-existing cardiovascular or cerebrovascular disease } & $1.45(1.24-1.70)$ & $<0.001$ & $1.79(1.48-2.15)$ & $<0.001$ & $1.64(1.39-1.93)$ & $<0.001$ \\
\hline \multicolumn{2}{|l|}{ Low ISCED (0-2) } & $1.27(1.12-1.45)$ & $<0.001$ & $1.36(1.15-1.60)$ & $<0.001$ & $1.36(1.18-1.56)$ & $<0.001$ \\
\hline \multicolumn{2}{|l|}{$\mathrm{HCY}>$ median } & $1.18(1.05-1.32)$ & 0.005 & $1.15(1.00-1.33)$ & 0.057 & I.IO (0.97-I.24) & 0.141 \\
\hline \multicolumn{2}{|l|}{ CRP $>3 \mathrm{mg} / \mathrm{L}$} & $1.12(1.00-1.26)$ & 0.052 & $1.19(1.03-1.38)$ & 0.018 & $1.22(1.08-1.38)$ & 0.002 \\
\hline \multicolumn{2}{|c|}{ GGT highest quartile } & $1.05(0.92-1.19)$ & 0.492 & $1.13(0.96-1.32)$ & 0.145 & $1.08(0.94-1.25)$ & 0.266 \\
\hline \multicolumn{2}{|c|}{ eGFR $<60 \mathrm{~mL} / \mathrm{min} / \mathrm{I} .73 \mathrm{~m}^{2}$} & $\mathrm{I} .04(0.89-1.22)$ & 0.624 & $1.06(0.88-1.28)$ & 0.554 & $1.18(1.00-1.38)$ & 0.051 \\
\hline \multicolumn{2}{|c|}{ Vitamin $D<50 \mathrm{nmol} / \mathrm{L}$} & $1.10(0.97-1.26)$ & 0.133 & $1.07(0.91-1.26)$ & 0.390 & $1.16(1.01-1.33)$ & 0.042 \\
\hline
\end{tabular}

Abbreviations: PAD, peripheral artery disease; LDL, Low density lipoprotein; BMI, body mass index; ISCED, International Standard Classification of Education; HCY, homocysteine; CRP, C-reactive protein; GGT, gamma-glutamyl transferase; eGFR, estimated glomerular filtration rate; $\mathrm{Cl}$, confidence interval; $\mathrm{N}$, number.

1.72 , 3rd), active smoking (1.79 vs $2.36,2 \mathrm{nd})$, diabetes (1.20 vs $1.33,2$ nd and $3 \mathrm{rd})$, pre-existing cardiovascular or cerebrovascular disease (1.45 vs $1.79,2 \mathrm{nd})$, and CRP (1.12 vs 1.22 , 3rd). Comparing risk factors between regression A (interpolation only) and regression B (with extension to whole observation period), vitamin D deficiency was more pronounced in regression method B. Here, characteristics of older patients which more frequently were lost to follow-up and are known to have lower vitamin D levels may play a role. Age $>75$ years, active smoking status, diabetes, pre-existing cardio or cerebrovascular disease, arterial hypertension, low ISCED (0-2), LDL $\geq 130 \mathrm{mg} / \mathrm{dL}$, and body mass index (BMI) $\geq 30 \mathrm{~kg} / \mathrm{m}^{2}$ were significant PAD predictors according to all three PAD definitions in the multivariable analysis.

\section{Comparison with Other Studies}

Most studies on PAD incidence and risk factors define PAD onset by a single ABI value $<0.9$. In a study on PAD incidence investigating the urban population in
Spain with a follow-up of 5 years, $12 \%$ of the 468 male subjects experienced PAD onset. ${ }^{7}$ This corresponded to an incidence rate of 23.8 events per 1,000 person years. Among subjects $>70$ years (an age group like our ABI cohort), the cumulative incidence for PAD onset was $22.4 \%$.

A study which investigated PAD incidence and risk factor association in subjects with type 2 diabetes ${ }^{10}$ found that $20.5 \%$ of the 1,479 participants experienced PAD onset during an average of 4.6 years of follow-up. The results of both studies are similar to our findings according to the PAD definition which also used a single ABI value $<0.9$ to determine PAD onset. According to our 1st PAD definition $24.1 \%(1,270$ of 5,269$)$ of the subjects experienced PAD onset.

The Limburg PAOD study, which investigated PAD incidence in 2,327 subjects from 18 general practice centers in the Netherlands ${ }^{11}$ with an average follow-up of 7.2 years, used two measurements to determine PAD onset. PAD was defined by an $\mathrm{ABI}<0.95$, measured twice in 
intervals of one week. In the age group $\geq 65$ years, men experienced 17.8 events per 1000 person years and women 22.9 events per 1,000 person years. This is comparable to the incidence rates according to our 2nd PAD definition that employed multiple $\mathrm{ABI}$ determinations.

Numerous studies showed a significant association between age and incident PAD. ${ }^{7,13-15}$ An especially high effect of age was displayed by the Limburg PAOD study with an OR of 4.0 (95\% CI 2.8 to 5.9) for subjects $\geq 65$ years. ${ }^{11}$ The Limburg PAOD study had the age category of 40-54 years as reference group, which may explain the high estimate.

With the help of the CALIBER program, the associations of cardiovascular disease and smoking ${ }^{13}$ as well as diabetes ${ }^{14}$ were investigated in over 1.9 million people each. Compared with never smoking, current smoking displayed a HR of 5.16 (95\% CI 4.80 to 5.54) for incident PAD. Diabetes showed a HR of 2.98 (95\% CI 2.76 to 3.22 ).

According to the REGICOR study, ${ }^{6}$ smoking was the most important predictor for incident PAD among subjects $>65$ years (OR $2.30,95 \%$ CI 1.27 to 4.16 ), while diabetes was the most relevant in patients $\leq 65$ years (OR 1.78, 95\% CI 1.17 to 2.72). This coincides with our findings as smoking was the strongest predictor for incident PAD. Diabetes had an especially great effect on PAD incidence according to our 2nd and 3rd PAD definitions.

While age and smoking were significantly associated with incident PAD in the ARTPER population cohort, ${ }^{12}$ diabetes was a significant predictor in the univariable model only. As shown by our findings, PAD definitions which used the single ABI method often displayed a lesser impact of risk factors. Thus, a possible explanation for diabetes not being significant in the multivariable analysis of the ARTPER population cohort may be the use of the single ABI method.

A recent work on incident symptomatic PAD and lipid concentrations in the population of the Women's Health Study (WHS) ${ }^{15}$ presented elevated LDL particle concentration (LDL-P) (HR 2.03, 95\% CI 1.14 to 3.59 for elevated total LDL-P), but not LDL cholesterol as significant predictors of incident PAD. In our work, in which we included the symptomatic as well as the asymptomatic component of PAD, elevated LDL levels were significantly associated with incident PAD according to all three PAD definitions.

An individual patient data meta-analysis with a total of 817,084 subjects and a median follow-up of 7.4 years investigated the association of impaired renal function and incident PAD using splines. ${ }^{16}$ With an eGFR of 95 $\mathrm{mL} / \mathrm{min} / 1.73 \mathrm{~m}^{2}$ as the reference value, the HR was 1.22 (95\% CI 1.14 to 1.30 ) for an eGFR of $45 \mathrm{~mL} / \mathrm{min} / 1.73 \mathrm{~m}^{2}$ and 2.06 (95\% CI 1.70-2.48) for an eGFR of $15 \mathrm{~mL} / \mathrm{min} /$ $1.73 \mathrm{~m}^{2}$. Their findings were different from ours, where the eGFR played a minor role, for the 3rd PAD definition a low eGFR (dichotomized at $60 \mathrm{~mL} / \mathrm{min} / 1.73 \mathrm{~m}^{2}$ ) was at the boundary for significance (HR 1.18, 95\% CI 1.00 to 1.38 ).

A work on PAD incidence, which also used data from the getABI study and defined PAD onset similar to our 2nd PAD definition except that intermittent claudication was disregarded for the symptomatic part, ${ }^{2}$ underlined the importance of many already well-known PAD risk factors and also investigated novel ones. While, analogous to our work, smoking, cardiovascular disease comorbidity, older age $>75$ years and diabetes were some of the most important predictors, low vitamin D levels and a low level of education expressed by an ISCED level of 0-3 were not significantly associated with incident PAD. In our work, low vitamin D levels were important PAD predictors according to our 3rd definition with a HR of 1.16 (95\% CI 1.01 to 1.33). Rethinking educational levels in the 5th and 6th decade of the last century, and considering level 3 as the most frequent, we now defined exposure to lower education as an ISCED level of 0-2 and found that it was significantly associated with incident PAD according to each of our three different PAD definitions. To the best of our knowledge, there are not any other large-scale works confirming the association of a low level of education and incident PAD.

\section{Strengths and Limitations}

With a total of 6,880 unselected study participants, this study has one of the largest cohorts addressing PAD in a primary care setting. The generalizability of results was improved by recruiting patients all over Germany by a total of 344 GPs with a simultaneous recruitment time of two weeks. Results provided by this primary care setting are more likely to be directly applicable to routine care compared to population-based cohorts. To the best of our knowledge, there are no other studies investigating and comparing PAD incidence rates and risk factor associations to a similar extent as our work using the three different PAD definitions.

A limitation of this study is the age requirement of $\geq 65$ years as an inclusion criterion. However, due to the 
demographic change, the results may gain even more relevance in the future.

Since the 3rd definition has not yet been implemented in any other studies on PAD incidence, this leads to a limited comparability with other works. Although, there was no external criterion to decide about the best approach, we argued for some overestimation of PAD incidence using the single ABI method for theoretical reasons.

\section{Conclusion}

Compared to PAD definitions which used regression methods and thus multiple ABI determination (2nd and 3rd PAD definition), the single ABI method (1st PAD definition) yielded a higher PAD incidence rate and ascribed many PAD risk factors a smaller importance. This was particularly noticeable for the risk factors older age, smoking, pre-existing cardiovascular or cerebrovascular disease. Also, the risk factor vitamin D deficiency would have been overlooked in this cohort without the 3 rd definition, which reflects the progress of PAD beyond the last ABI measurement. Further, a low level of education was a significant predictor of incident PAD according to all three PAD definitions.

Our findings suggest that using the single ABI method for defining PAD onset may lead to overestimation in assessing incident PAD in epidemiological studies. Since regression methods can be interpreted as a correction for a randomly low ABI value while estimating the time to PAD onset, the evaluation of PAD risk factors via proportional hazards might also be more precise and the check for trends in ABI values might be helpful. Nevertheless, without an external criterion the best method for clinical use cannot be determined and more research is needed, which, eg, compares discriminatory capabilities of various PAD definitions for hard endpoints, such as mortality.

\section{Acknowledgments}

Special thanks go to the participating general practitioners and angiologists as well as all the participants of the getABI trial. We acknowledge support by the Open Access Publication Funds of the Ruhr University Bochum.

\section{Disclosure}

Prof. Dr. Hans J Trampisch reports grants from SanofiAventis GmbH, Berlin, and from German Federal Ministry of Education and Research, during the conduct of the study. The authors report no other conflict of interest in this work.

\section{References}

1. Song P, Rudan D, Zhu Y, et al. Global, regional, and national prevalence and risk factors for peripheral artery disease in 2015: an updated systematic review and analysis. Lancet Glob Health. 2019;7 (8):e1020-e1030. doi:10.1016/S2214-109X(19)30255-4

2. Krause D, Burghaus I, Thiem U, et al. The risk of peripheral artery disease in older adults - seven-year results of the getABI study. Vasa. 2016;45:403-410. doi:10.1024/0301-1526/a000556

3. Xu D, Zou L, Xing Y, et al. Diagnostic value of ankle-brachial index in peripheral arterial disease: a meta-analysis. Can J Cardiol. 2013;29:492-498. doi:10.1016/j.cjca.2012.06.014

4. Diehm C, Allenberg JR, Pittrow D, et al. Mortality and vascular morbidity in older adults with asymptomatic versus symptomatic peripheral artery disease. Circulation. 2009;120:2053-2061. doi:10.1161/CIRCULATIONAHA.109.865600

5. Lawall H, Diehm C, Hoffmann U, et al. [Update PAVK: epidemiology, comorbidity and prognosis of peripheral arterial obstructive disease]. Dtsch Med Wochenschr. 2015;140:1798-1802. (German). doi:10.1055/s-0041-107064

6. Velescu A, Clara A, Peñafiel J, et al. Peripheral arterial disease incidence and associated risk factors in a Mediterranean population-based cohort. The REGICOR Study. Eur J Vasc Endovasc Surg. 2016;51:696-705. doi:10.1016/j.ejvs.2015.12.045

7. Merino J, Planas A, Elosua R, et al. Incidence and risk factors of peripheral arterial occlusive disease in a prospective cohort of 700 adult elderly men followed for 5 years. World $J$ Surg. 2010;34:1975-1979. doi:10.1007/s00268-010-0572-7

8. Holland-Letz T, Endres HG, Biedermann S, et al. Reproducibility and reliability of the ankle-brachial index as assessed by vascular experts, family physicians and nurses. Vasc Med. 2007;12:105-112. doi:10.1177/1358863X07077281

9. getABI Study Group. getABI: German epidemiological trial on ankle brachial index for elderly patients in family practice to detect peripheral arterial disease, significant marker for high mortality. Vasa. 2002;31:241-248. doi:10.1024/0301-1526.31.4.241

10. Althouse AD, Abbott JD, Forker AD, et al. Risk factors for incident peripheral arterial disease in type 2 diabetes: results from the Bypass Angioplasty Revascularization Investigation in type 2 Diabetes (BARI 2D) Trial. Diabetes Care. 2014;37(5):1346-1352. doi:10.2337/dc132303

11. Hooi JD, Kester AD, Stoffers HE, et al. Incidence of and risk factors for asymptomatic peripheral arterial occlusive disease: a longitudinal study. Am J Epidemiol. 2001;153:666-672. doi:10.1093/aje/ 153.7.666

12. Alzamora MT, Forés R, Pera G, et al. Incidence of peripheral arterial disease in the ARTPER population cohort after 5 years of follow-up. BMC Cardiovasc Disord. 2016;16:8. doi:10.1186/ s12872-015-0170-6

13. Pujades-Rodriguez M, George J, Shah AD, et al. Heterogeneous associations between smoking and a wide range of initial presentations of cardiovascular disease in 1937360 people in England: lifetime risks and implications for risk prediction. Int J Epidemiol. 2015;44:129-141. doi:10.1093/ije/dyu218 
14. Shah AD, Langenberg C, Rapsomaniki E, et al. Type 2 diabetes and incidence of cardiovascular diseases: a cohort study in 1.9 million people. Lancet Diabetes Endocrinol. 2015;3:105-113. doi:10.1016/ S2213-8587(14)70219-0

15. Aday AW, Lawler PR, Cook NR, et al. Lipoprotein particle profiles, standard lipids, and peripheral artery disease incidence. Circulation. 2018;138:2330-2341. doi:10.1161/CIRCULATIONAHA.118.035432
16. Matsushita K, Ballew SH, Coresh J, et al. Measures of chronic kidney disease and risk of incident peripheral artery disease: a collaborative meta-analysis of individual participant data. Lancet Diabetes Endocrinol. 2017;5:718-728. doi:10.1016/S2213-8587(17) 30183-3

\section{Publish your work in this journal}

Vascular Health and Risk Management is an international, peerreviewed journal of therapeutics and risk management, focusing on concise rapid reporting of clinical studies on the processes involved in the maintenance of vascular health; the monitoring, prevention and treatment of vascular disease and its sequelae; and the involvemen of metabolic disorders, particularly diabetes. This journal is indexed on PubMed Central and MedLine. The manuscript management system is completely online and includes a very quick and fair peerreview system, which is all easy to use. Visit http://www.dovepress. com/testimonials.php to read real quotes from published authors. 